\title{
Uniportal video-assisted thoracoscopic surgery and robot- assisted thoracoscopic surgery are feasible approaches with potential advantages in minimally invasive mediastinal lesions resection
}

\author{
Liping Zeng", Weidong Wang ${ }^{\#}$, Jia Han, Linhai Zhu, Jiangang Zhao, Zhengliang Tu \\ Department of Thoracic Surgery, The First Affiliated Hospital, School of Medicine, Zhejiang University, Hangzhou, China \\ Contributions: (I) Conception and design: L Zeng, W Wang; (II) Administrative support: Z Tu; (III) Provision of study materials or patients: J Han, \\ L Zhu; (IV) Collection and assembly of data: L Zeng, W Wang, J Zhao; (V) Data analysis and interpretation: L Zeng, W Wang; (VI) Manuscript \\ writing: All authors; (VII) Final approval of manuscript: All authors. \\ \#These authors contributed equally to this work. \\ Correspondence to: Zhengliang Tu, PhD, MD. Department of Thoracic Surgery, The First Affiliated Hospital, School of Medicine, Zhejiang \\ University, No. 79 Qingchun Road, Hangzhou 310003, China. Email: drtuzhengliang@zju.edu.cn.
}

Background This study aims to identify the feasibility of uniportal video-assisted thoracoscopic surgery
(VATS) and robot-assisted thoracoscopic surgery (RATS) compared with multiportal VATS in the resection of mediastinal lesions.

Methods: Patients who underwent mediastinal lesion resection were enrolled and allocated to the uniVATS, tri-VATS, and RATS groups according to the surgical approach. Propensity score-matched (PSM) analysis was performed between the VATS and RATS groups as well as the uni-VATS and tri-VATS groups. The operative and recovery parameters were compared.

Results: Totally, 274 patients were enrolled. There was no difference in the operative parameters among the groups. Compared with multiportal VATS, uniportal VATS and RATS had a significantly shorter chest tube placement time $(2.43 \pm 0.88$ vs. $1.78 \pm 1.22$ vs. $2.21 \pm 1.11$ days, $\mathrm{P}<0.001)$ and hospital length of stay (LOS) $(4.07 \pm 1.75$ vs. $3.27 \pm 1.05$ vs. $3.62 \pm 1.21$ days, $\mathrm{P}=0.001)$ without increasing the incidence rate of complications (5.6\% vs. $7.2 \%$ vs. $5.7 \%, \mathrm{P}=0.864$ ). After PSM, the RATS group showed a significantly lower unplanned conversion rate than the VATS group $(0.0 \%$ vs. $8.2 \%, \mathrm{P}=0.041)$, while the uni-VATS group had a shorter chest tube placement time $(1.83 \pm 1.20$ vs. $2.35 \pm 0.86$ days, $\mathrm{P}=0.013)$ and hospital LOS $(3.23 \pm 1.03$ vs. $3.95 \pm$ 2.00 days) than the tri-VATS group.

Conclusions: Compared with multiportal VATS, uniportal VATS and RATS are technically safe and feasible with potential advantages for mediastinal lesion resection.

Keywords: Mediastinal lesions; minimally invasive surgery; video-assisted thoracoscopic surgery (VATS); uniportal video-assisted thoracoscopic surgery (uniportal VATS); robot-assisted thoracoscopic surgery (RATS)

Submitted May 30, 2020. Accepted for publication Sep 24, 2020.

doi: $10.21037 / g s-20-536$

View this article at: http://dx.doi.org/10.21037/gs-20-536

^ ORCID: 0000-0002-1168-8803. 


\section{Introduction}

The mediastinal lesions, including thymoma, Thymic hyperplasia, etc., are common diseases. Generally, surgical treatment is the first choice in clinical practice, and most patients have a good prognosis. Traditional surgical approaches include sternotomy, lateral thoracotomy, and minimally invasive surgery. Emerging as a minimally invasive approach, video-assisted thoracoscopic surgery (VATS) has been widely used in the treatment of mediastinal tumors. A large series of studies have proven that VATS has unique advantages over open techniques due to its advantages of minimal trauma, few complications, and rapid postoperative recovery (1-3).

With the improvement of thoracoscopic instruments and the advancement of surgical techniques, incisions have been gradually reduced from multiportal to uniportal, pursuing reduced postoperative discomfort caused by multiple incisions and enhanced recovery after surgery (4). Compared with conventional multiportal VATS, uniportal VATS has unique characteristics in terms of further improved cosmesis, an optimized visual field, reduced postoperative pain, and improved recovery (5). In recent years, it has been widely implemented, and a large series of experiences with promising outcomes have been reported (6). Besides, since the first case of mediastinal tumor resection by the da Vinci surgical system in 2001, robot-assisted thoracoscopic surgery (RATS) for mediastinal tumor has attracted more attention, with good clinical efficacy and safety (7). Uniportal VATS and RATS appear to have less invasiveness and better recovery than multiportal VATS.

Several studies have been conducted to compare minimally invasive thoracic surgery to open approaches; however, there are few comparisons among minimally invasive approaches. Thus, we conducted this study to analyze the safety, feasibility, and short-term outcomes of uniportal VATS and RATS compared with multiportal VATS in patients who underwent mediastinal lesion resection.

We present the following article in accordance with STROBE reporting checklist (available at http://dx.doi. org/10.21037/gs-20-536).

\section{Methods}

\section{Patients}

The study was a retrospective study and was designed to compare the safety, feasibility, and short-term outcomes of uniportal VATS and RATS compared with multiportal VATS in patients who underwent mediastinal lesion resection. The study was conducted in accordance with the Declaration of Helsinki (as revised in 2013). This study was approved by the ethics committee of the First Affiliated Hospital, School of Medicine, Zhejiang University, the reference number is 2019-748-1 and individual consent for this retrospective analysis was waived. Data from 298 patients with mediastinal lesions who underwent minimally invasive R0 resection surgery were initially collected. Patients were divided into 3 groups according to the surgical approach: the uniportal VATS (uni-VATS group), multiportal VATS (tri-VATS group), and the RATS group. All the patients were eligible for minimally invasive surgery, and there was no obvious invasion of greater vessels, no history of mediastinal surgery, no severe systematic diseases, and no distant metastases in any patient. Patients who underwent palliative surgery, biopsy, neoadjuvant therapy, or simultaneous resection of the mediastinal tumors with pulmonary nodules were excluded from the study (Figure 1).

A propensity score-matched (PSM) comparative analysis was performed to balance the nonrandom baseline characteristics among the groups. We adjusted for potential differences between the VATS group (both uni-VATS and tri-VATS group) and RATS group with 1:1 matching. The same adjustment was also performed in the VATS group internally. We produced a propensity score for the matched groups using logistic regression based on the patient's age, sex, tumor location, tumor pathology, myasthenia symptom and tumor size with a caliper setting of 0.02 .

\section{Surgical approach}

Generally, the surgery was performed under general anesthesia. The patient was positioned in a lateral decubitus position or 30-degree semisupine position with the ipsilateral axilla exposed.

After probing the chest, energy equipment, such as a harmonic scalpel, was used to carefully dissociate the border of the lesion and completely resect the entire lesion within the anatomic landmark of the phrenic nerve, internal mammary vein, brachiocephalic vein, pericardium and sternum. The nourishing vein branches were divided with extra caution and clamped with hem-o-lock or titanium clips when necessary. Great attention was paid to exposing and protecting the left brachiocephalic vein in cases of injury and bleeding. For thymoma with myasthenia, a 


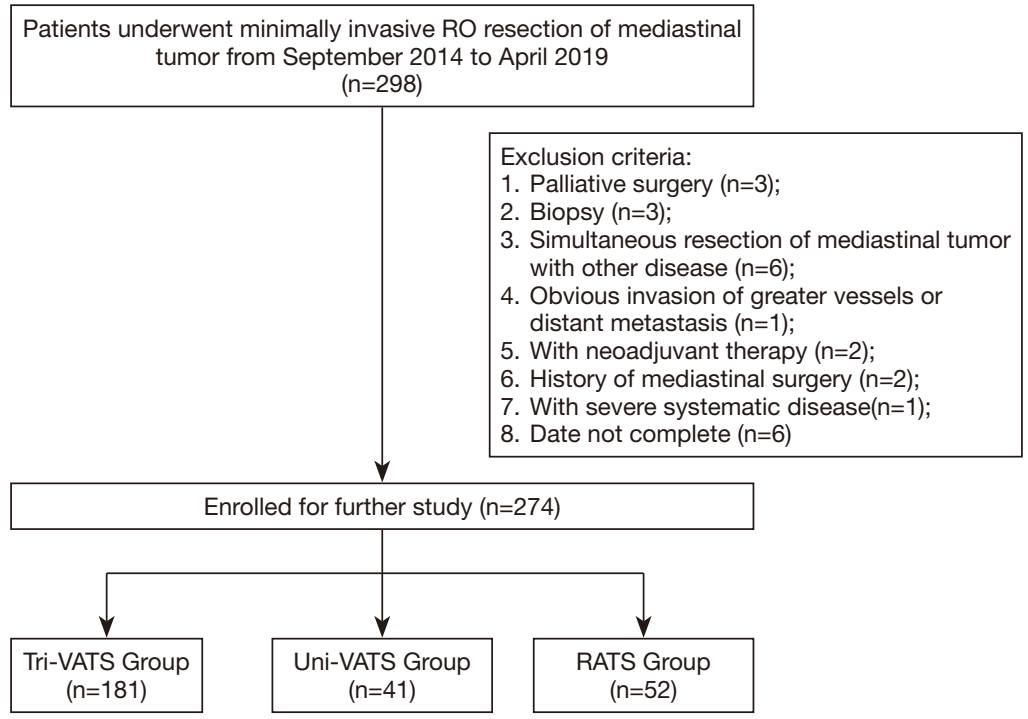

Figure 1 Flowchart of this study. VATS, video-assisted thoracoscopic surgery; RATS, robot-assisted thoracic surgery.

complete thymectomy with surrounding fat dissection was performed. The incision could be properly extended when a giant tumor was retrieved.

For multiport VATS, a typical 3-port approach was used, with one observation port and two operation ports. A $1-\mathrm{cm}$ observation port was made in the 5 th intercostal space at the mid-axillary line. The other 2 ports were placed in the 3rd intercostal space, at the anterior axillary line, and in the 5 th intercostal space, at the midclavicular line.

For uniportal VATS, a single incision was preferably made at the 4th intercostal space (3rd or 5 th for select patients) in the anterior axillary line or the mid-axillary line, depending on the location of the mediastinal tumor. The incision was approximately $3-4 \mathrm{~cm}$ in length and retracted with a wound protector. The surgery was performed with the cooperation of curved suction and the harmonic scalpel or electrocautery hook.

For RATS, a 3-port approach was performed. For patients whose tumor is located in the front or middle mediastinum, a $1 \mathrm{~cm}$ observation port was made in the 5 th intercostal space at the mid-axillary line. One of the assistant ports was placed in the 3 rd intercostal space at the anterior axillary line while another one was placed in the 5th intercostal space at the midclavicular line. And for those with tumor at the post-mediastinum, the observation port was made in the 7 th intercostal space and the other 2 assistant ports were placed in the 5 th intercostal space, at the anterior axillary line, and in the 7th intercostal space, at the midclavicular line.

\section{Postoperative pain and analgesia}

Intravenous patient-controlled analgesia (hydromorphone $6 \mathrm{mg}$ and flurbiprofen $200 \mathrm{mg}$ ) and paravertebral blockade $(\mathrm{PB})$ of $0.4 \%$ ropivacaine $20 \mathrm{~mL}$ were used to control postoperative pain. The pain of patients was evaluated at 8 a.m. postoperative day one using the visual analog score.

\section{Outcome measures}

The duration of operation, intraoperative blood loss, incidence of unplanned thoracotomy, chest tube drainage time, postoperative complications (including pleural effusion or pneumothorax, fever, pulmonary thromboembolism (PTE), chylothorax, and arrhythmia), postoperative pain and hospital length of stay (LOS) were observed and compared among the groups. A hospital LOS $\geq 7$ days, intraoperative blood loss $\geq 200 \mathrm{~mL}$, conversion to thoracotomy and complications were defined as adverse outcomes.

All the clinical data were collected from the electronic medical records system.

\section{Statistical analysis}

Categorical variables were calculated using the $\chi^{2}$ test and Fisher's exact test, while continuous variables were analyzed using the $t$-test, Mann-Whitney U-test, ANOVA test or Kruskal-Wallis test. Logistic regression was performed to 
Table 1 Clinicopathological characteristics of the study population

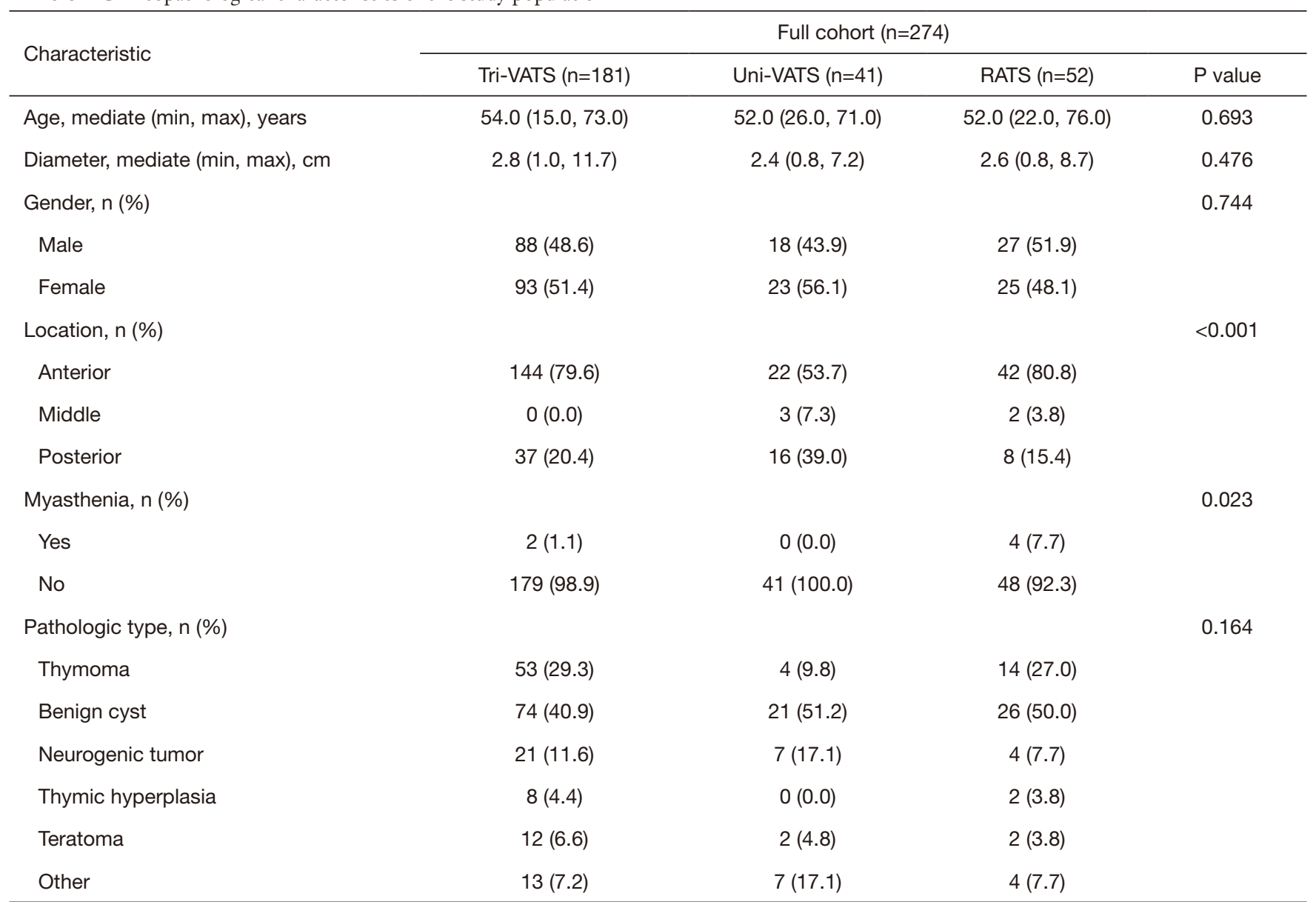

CM, centimeter; Max, maximum; Min, minimum; RATS, robot-assisted thoracic surgery; SD, standard deviation; Tri-VATS, triportal videoassisted thoracoscopic, Uni-VATS, uniportal video-assisted thoracoscopic.

identify risk factors for adverse outcomes. For all analyses, two-sided $\mathrm{P}$ values $<0.05$ were considered statistically significant. Odds ratios (ORs), 95\% confidence intervals (CIs), $\mathrm{P}$ values and PSM analyses were performed and determined by using SPSS 22.0 software (IBM, Armonk, NY), and figures were drawn by using GraphPad Prism 5.0 software (GraphPad Software, La Jolla, CA).

\section{Results}

\section{Study population}

A total of 274 patients were included and analyzed in the study: 181 patients in the multiportal VATS (tri-VATS) group, 41 patients in the uniportal VATS (uni-VATS) group and 52 patients in the RATS group. Patients in the uni-VATS group had more posterior mediastinal diseases, while the RATS group had more patients with symptoms of myasthenia. There was no significant difference in age, tumor diameter, sex, or pathologic type among the 3 groups (Table 1).

\section{Operability and intraoperative safety}

There were no differences in the duration of operation among the tri-VATS, uni-VATS and RATS groups $(74.14 \pm 34.91,69.18 \pm 32.91$ and $78.08 \pm 32.58$ minutes, respectively, $\mathrm{P}=0.154$ ) (Figure $2 A$ ). The uni-VATS group had the lowest volume of intraoperative blood loss at $18.08 \pm 16.73 \mathrm{~mL}$, but the tendency was not statistically significant $(\mathrm{P}=0.077)$ (Figure $2 B)$. A total of $3.9 \%$ of patients in the tri-VATS group and $4.9 \%$ of patients in the uni-VATS group suffered from unplanned thoracotomy, 

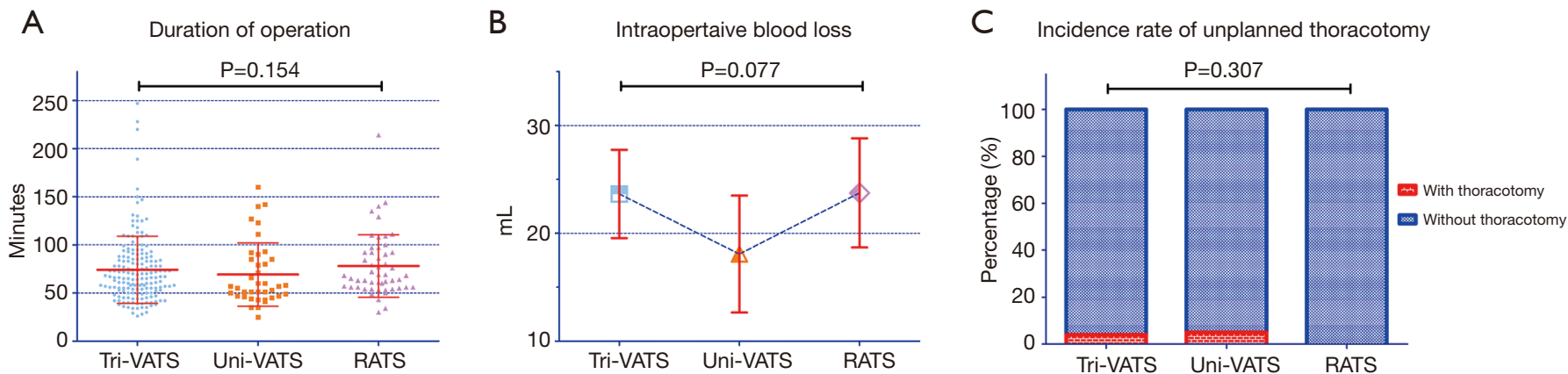

Figure 2 Comparison of operative parameters among the 3 groups. (A) Duration of operation in the 3 groups; (B) intraoperative blood loss volume of the 3 groups; (C) incidence rate of unplanned conversion to thoracotomy in the 3 groups. VATS, video-assisted thoracoscopic surgery; RATS, robot-assisted thoracic surgery.

while the ratio was $0.0 \%$ in the RATS group. However, the incidence rate of unplanned thoracotomy was not significantly different $(\mathrm{P}=0.307)$ (Table 2, Figure $2 C)$.

\section{Postoperative outcomes}

The mean duration of chest tube placement in the uniVATS group was $1.78 \pm 1.22$ days, which was significantly shorter than that in the tri-VATS group and RATS group $(\mathrm{P}<0.001, \mathrm{P}=0.030$; respectively). However, the difference between the RATS group and the tri-VATS group was not significant $(\mathrm{P}=0.134)$ (Table 2, Figure $3 A$ ).

The total incidence rate of all complications was $5.6 \%$, $7.2 \%$, and $5.7 \%$ in the tri-VATS group, uni-VATS group, and RATS group, respectively, but the differences were not statistically significant $(\mathrm{P}=0.864)$ (Figure $3 B)$. After a subgroup analysis, the incidence rate of each complication was also not different among the groups (Table 2). Patients in the RATS group had a higher proportion of degree I pain, though the difference was not significant enough (Table 2).

The tri-VATS group had the longest hospital LOS at $4.07 \pm 1.75$ days, while the uni-VATS group had the shortest LOS at $3.27 \pm 1.05$ days $(\mathrm{P}=0.006)$. However, the hospital LOS in the RATS group was $3.62 \pm 1.21$ days, a difference that was of marginal significance from the tri-VATS group and the uni-VATS group $(\mathrm{P}=0.061$ and $\mathrm{P}=0.085$, respectively) (Table 2, Figure 3C).

The total expense was similar between the uni-VATS group and the tri-VATS group $(\mathrm{P}=0.233)$. However, the total expense of both VATS groups was much lower than that of the RATS group (Table 2).

\section{Risk factors for adverse outcomes}

According to the definition of adverse outcomes, 31 patients suffered it. After logistic regression, only the diameter of the tumor was an independent risk factor for adverse outcomes. Compared with tri-VATS, both uni-VATS and RATS showed no statistical significance, indicating that the different types of surgical approaches were not independent risk factors for adverse outcomes (Table S1).

\section{Comparison after PSM between the VATS group and the RATS group}

After PSM analysis, 98 (49 pairs) patients were enrolled, and the baseline characteristics were homogeneous (Table S2). Except patients with unplanned thoracotomy, the duration of operation was not significantly different between the two groups $(\mathrm{P}=0.083)$, while the volume of intraoperative blood loss was higher in the RATS group. However, the incidence rate of unplanned thoracotomy in the VATS group was significantly higher $(8.2 \%$ vs. $0.0 \%, \mathrm{P}=0.041$ ) (Table 3 , Figure 4).

The RATS group had a significantly higher total expense and lower postoperative pain, but the chest tube placement time, hospital LOS and incidence rate of complications were similar to those of the VATS group (Table 3, Figure 4). After logistic regression, although the RATS approach showed the potential to be a protective factor for adverse outcomes, the tendency was not statistically significant (OR, 0.456; 95\% CI, 0.128-1.626, P=0.226) (Table S3). 
Table 2 Comparisons of the intraoperative and postoperative parameters among the 3 groups

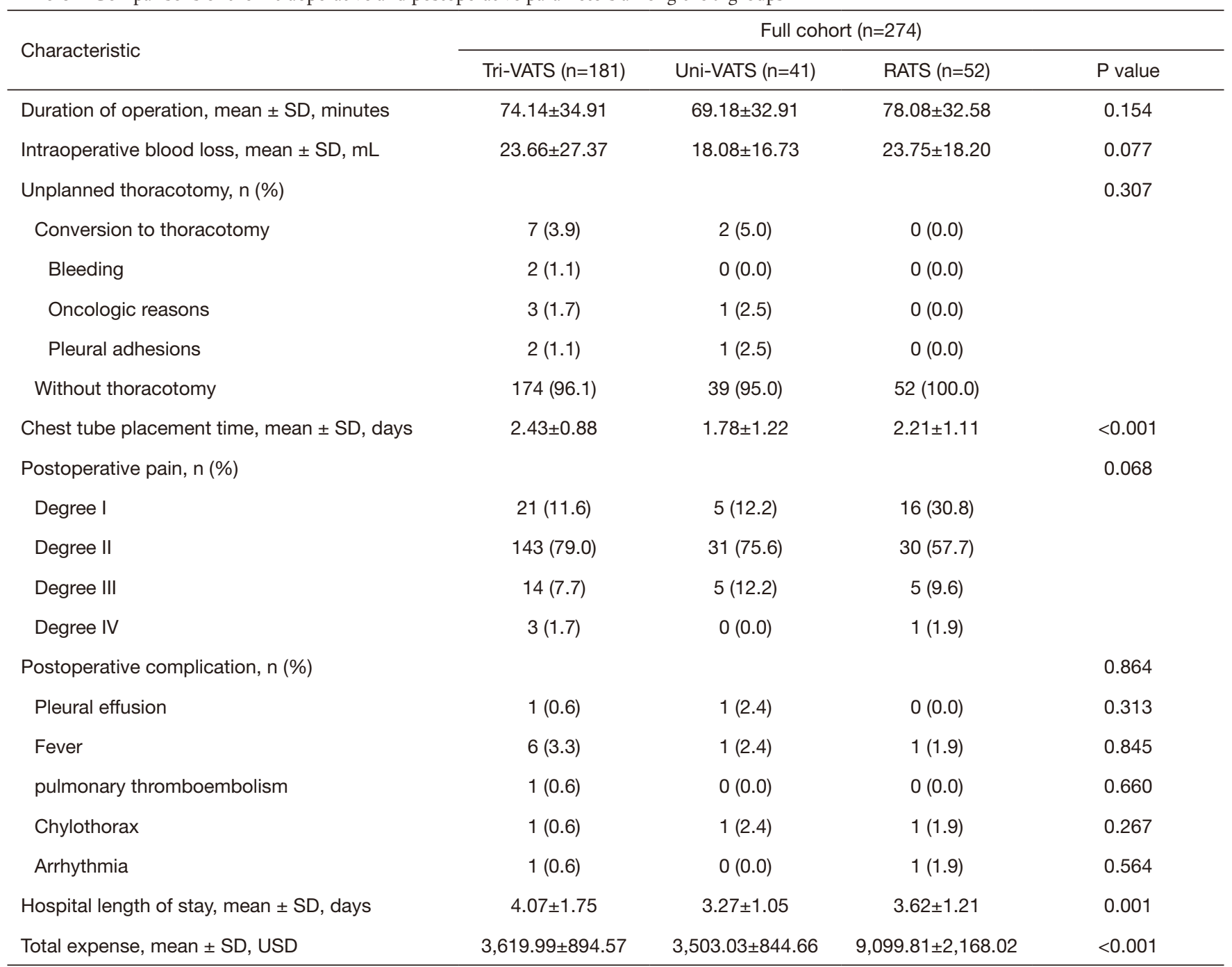

$\mathrm{mL}$, milliliter; RATS, robot-assisted thoracic surgery; SD, standard deviation; USD, USA dollar; VATS, video-assisted thoracoscopic.

A Chest tube placement time (days)

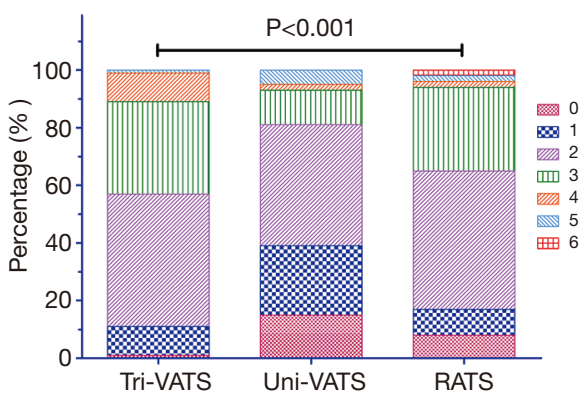

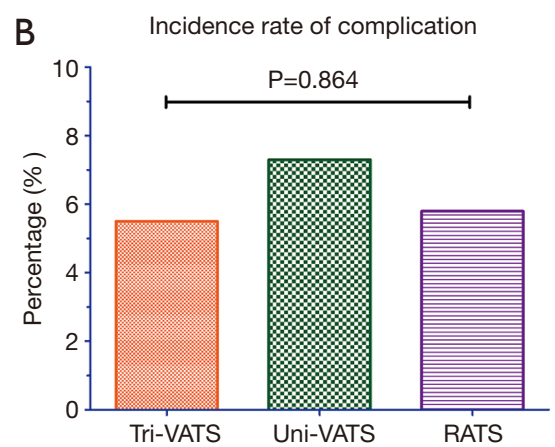

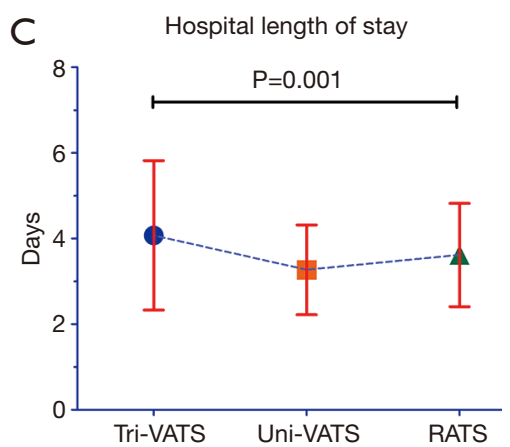

Figure 3 Comparison of postoperative recovery characteristics among the 3 groups. (A) Chest tube placement time in the 3 groups; (B) total incidence rate of all the postoperative complications in the 3 groups; (C) postoperative hospital length of stay in the 3 groups. VATS, videoassisted thoracoscopic surgery; RATS, robot-assisted thoracic surgery. 


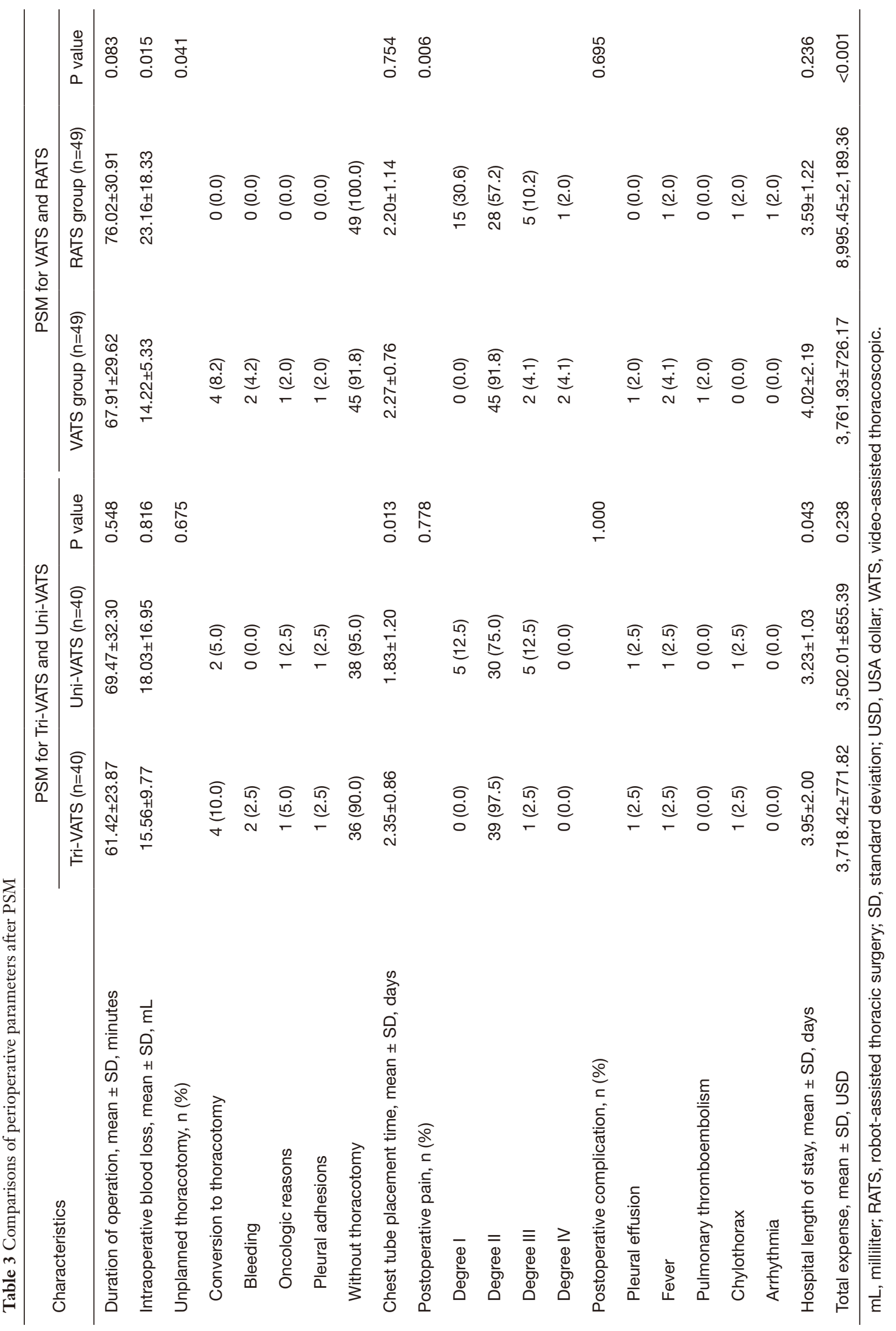



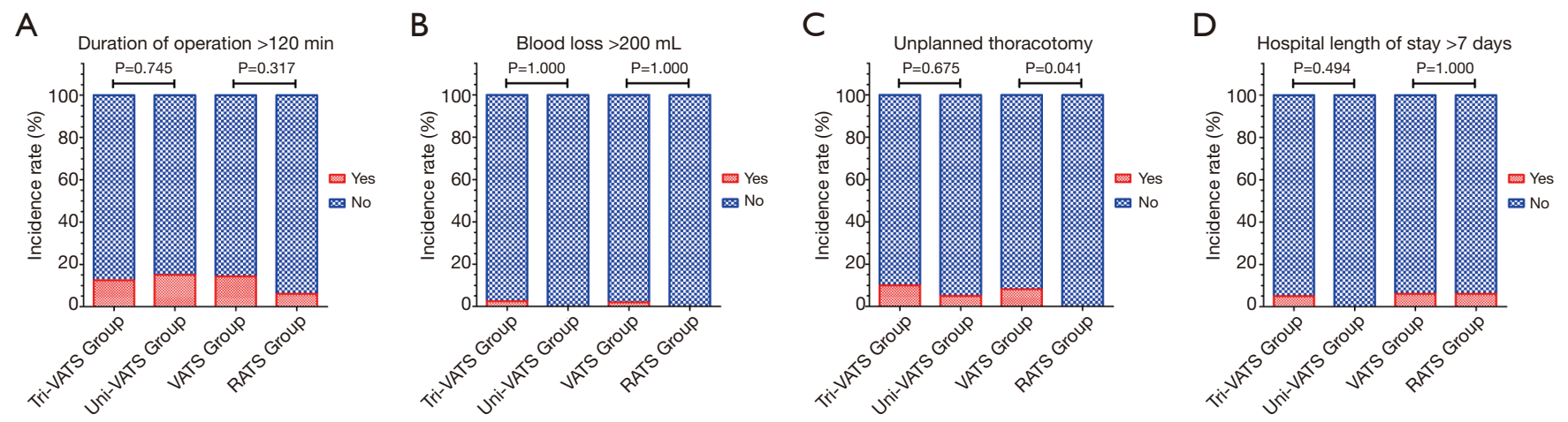

Figure 4 Comparison of perioperative parameters after PSM. (A) Incidence rate of surgical duration more than 120 minutes; (B) incidence rate of intraoperative blood loss more than $200 \mathrm{~mL}$; (C) incidence rate of unplanned conversion to thoracotomy; (D) incidence rate of hospital length of stay more than 7 days. mL, milliliter; VATS, video-assisted thoracoscopic surgery; RATS, robot-assisted thoracic surgery.

\section{Comparison after PSM between the uni-VATS group and the tri-VATS group}

A total of 80 (40 pairs) patients were enrolled in this PSM cohort, and the baseline characteristics were also homogeneous (Table S2). The chest tube placement time and hospital LOS were significantly shorter in the uniVATS group than in the tri-VATS group $(\mathrm{P}=0.013, \mathrm{P}=0.043$; respectively). No difference was observed in other operative or postoperative parameters (Table 3, Figure 4). Uni-VATS also showed the potential to be a protective factor for adverse outcomes, but the tendency was not significant (OR, 0.673; 95\% CI, 0.194-2.333, P=0.533) (Table S4).

\section{Discussion}

Mediastinal lesions are a common disease in thoracic surgery. Generally, surgical treatment is the first choice, and most patients have a good prognosis. Lewis et al. first applied VATS to remove mediastinal cysts in 1992, and since then, the minimally invasive approaches have been widely applied in the treatment of mediastinal diseases $(3,8)$. Several studies have proved that minimally invasive thoracic surgery was non-inferior to open approaches; however, the comparisons of operative parameters and recovery outcomes among minimally invasive approaches are few. In this study, we demonstrated that the resection of mediastinal disease was safe and feasible through the VATS or RATS approach. Compared to VATS, RATS had a lower incidence rate of unplanned thoracotomy and significantly lower postoperative pain, and the advantage was more significant after PSM. Furthermore, the postoperative recovery of uniportal VATS was superior to that of multiportal VATS in terms of the hospital LOS and duration of chest tube drainage without increasing the operative and postoperative complications.

Increasing evidence has preliminarily confirmed that the effect of minimally invasive surgery in tumor resection is similar to that of traditional thoracotomy with minimization of invasiveness caused by the operation (9). In addition, a series of studies reported the technical benefits of minimally invasive surgery over open surgery, with a lower estimated blood loss, chest tube duration, and hospital length of stay (10). However, traditional thoracoscopic surgery has certain limitations. First, the surgery requires 3-4 ports, resulting in multiple scars and chronic intercostal pain. Second, there are some inherent shortcomings of the technology, such as the twodimensional field, the insufficient processing capacity in a narrow space and the difficulty of sutures (11). During the past decade, thoracic surgeons have continued to reduce the invasiveness of surgery and achieved two milestones of minimally invasive thoracic surgery: uniportal VATS and RATS.

Uniportal VATS was pioneered by Rocco for wedge pulmonary resections in 2004 and was gradually extended by Dr. Diego Gonzalez-Rivas for other thoracic operations $(12,13)$. Compared with conventional multiportal VATS, uniportal VATS has unique characteristics in terms of further improvements in cosmesis, an optimized visual field, reduced postoperative pain and a better quality of life for patients. Currently, uniportal VATS is well adopted for the resection of mediastinal disease (5). The da Vinci robotic surgery system has unique advantages, such as 
excellent 3-dimensional vision, manual tremor filtering, and 7 degrees of freedom (14). Yoshino et al. first reported the resection of mediastinal tumors using the da Vinci surgical system. Currently, robotic surgery for mediastinal disease is widely applied, with good clinical efficacy and safety. Moreover, robotic surgery has revealed unique superiority while dealing with locally invasive disease (pericardium, greater vessel involvement) and tumors in narrow spaces (cupula pleurae) (7).

Several studies have been conducted to compare the safety and efficacy among different approaches. Buentzel et al. conducted a meta-analysis comparing RATS thymectomy and VATS thymectomy indirectly. The results showed that the approaches were equivalent in terms of the conversion rate, operation time and length of hospitalization, but there was a slightly greater blood loss in the RATS group (15). Qian et al. retrospectively compared the effect of extended thymectomy in the treatment of early-stage thymomas in 123 patients under RATS, VATS, and median sternotomy approaches. The results showed that minimally invasive surgery (including RATS and VATS) was feasible and safe for the resection of early-stage thymomas compared with median sternotomy. Compared with VATS, RATS showed a better minimally invasive effect, with a shorter drainage time, reduced drainage volume, and shorter hospital stays (16). O'Sullivan et al. conducted a systematic review of robotic versus open and VATS approaches for thymectomy. The results showed that robotic thymectomy was superior to open surgery and comparable to the VATS approach (17). In our study, although the expense of RATS was higher, this approach had better safety with a lower incidence rate of unplanned thoracotomy than VATS. Besides, robotic surgery also contributed greatly to the ease of patients' postoperative pain. The advantage was more significant after PSM.

Uniportal VATS has been increasingly adopted in thoracic surgery with the advantages of less invasiveness and a faster recovery. Several studies have reported the feasibility of uniportal VATS for mediastinal tumors resection. Rocco et al. showed that uniportal VATS could be effectively used to achieve a diagnosis of mediastinal nodes (18). Akter's study pointed out that, compared with multiport VATS, uniportal VATS could offer less postoperative pain without increasing the risk of operation and in-hospital stay (19). In 2017, Gonzalez-Rivas et al. reported a case of the successful removal of a giant thymoma by uniportal VATS in a patient who refused open surgery, which showed that single-port thoracoscopic excision of mediastinal tumors represents a safe and effective surgical option even for patients with giant tumors (20). Recently, Deng et al. indicated that uniportal VATS for resection of mediastinal disease is technically safe and feasible and has comparable operative and postoperative parameters with multiportal VATS (21). The results of our study showed that uniportal VATS had comparable operative parameters and treatment costs to multiportal VATS. In addition, patients who underwent uniportal VATS compared to other approaches showed enhanced recovery, and the advantage still existed after PSM.

It has been well established that surgeons should not simply pursue smaller and fewer incisions instead of taking safety as the primary concern. Therefore, in our study, the duration of operation, intraoperative blood loss and unplanned conversion to open surgery were considered to be critical parameters for evaluating the safety of the different surgical approaches. The results indicated that there was no significant difference in these 3 aspects among the uniportal and multiportal VATS and RATS groups. However, after PSM, the RATS group showed a reduced conversion rate compared to the VATS group, which may indicate that the RATS approach is safer. Postoperative parameters were also compared to evaluate the recovery and cost effect. There was no significant difference in terms of the incidence of complications. However, the patients who underwent uniportal VATS had a significantly reduced hospital LOS and chest tube placement time. Compared to multiportal VATS, uniportal VATS still had these advantages even after PSM. This phenomenon proved the advantages in terms of postoperative recovery of uniportal VATS over traditional multiportal approaches. The total expense of the uniportal and multiportal VATS approaches was similar, while RATS had a significantly higher treatment cost, which may impede the popularization of this novel technique. Thus, for patients whose tumors are located in the anterior, middle or posterior mediastinum, if uniport VATS was technically feasible for surgeons, this approach should be preferentially considered. because this approach has fewer scars, further improvements in cosmesis, better postoperative recovery without increasing the operation risk and expense. However, for patients whose lesions located in the superior mediastinum or other locations where the operation space was narrow as well as having pleural adhesions or unexpected tumor invasion of the surrounding structures, RATS should be recommended if technically possible and economically affordable as RATS has an optimized visual field, and the da Vinci system could enable surgeons to carry out more flexible and delicate 
actions which can improve the safety of the operations and avoid unplanned thoracotomy.

Our study also has several limitations. First, the biases of retrospective studies in terms of patient selection and operation performance were unavoidable. Second, the relatively small sample size of the uniportal VATS group and RATS group may have resulted in statistical bias. Third, the long-term prognosis outcomes were not compared and need to be further studied. Multicenter randomized control trials should be further conducted to clarify the role of these 3 approaches in the treatment of mediastinal disease.

\section{Conclusions}

Compared with multiportal VATS, uniportal VATS and RATS for mediastinal disease resection are feasible and effective. The RATS approach showed non-inferior postoperative outcomes with better intraoperative safety than the VATS approach. Additionally, the uniportal VATS approach revealed advantages in facilitating recovery over the multiportal VATS approach. Both RATS and uniportal VATS could be considered useful substitutions for the conventional minimally invasive technique for the mediastinal disease.

\section{Acknowledgments}

Funding: This work was supported by National Key R\&D Program of China (2017YFC0113500) and Major Science and Technology Projects of Zhejiang Province (2014C03032).

\section{Footnote}

Reporting Checklist: The authors have completed the STROBE reporting checklist. Available at http://dx.doi. org/10.21037/gs-20-536

Data Sharing Statement: Available at http://dx.doi. org/10.21037/gs-20-536

Peer Review File: Available at http://dx.doi.org/10.21037/gs20-536

Conflicts of Interest: All authors have completed the ICMJE uniform disclosure form (available at http://dx.doi. org/10.21037/gs-20-536). The authors have no conflicts of interest to declare.
Ethical Statement: The authors are accountable for all aspects of the work in ensuring that questions related to the accuracy or integrity of any part of the work are appropriately investigated and resolved. The study was conducted in accordance with the Declaration of Helsinki (as revised in 2013). This study was approved by the ethics committee of the First Affiliated Hospital, School of Medicine, Zhejiang University (2019-748-1) and individual consent for this retrospective analysis was waived.

Open Access Statement: This is an Open Access article distributed in accordance with the Creative Commons Attribution-NonCommercial-NoDerivs 4.0 International License (CC BY-NC-ND 4.0), which permits the noncommercial replication and distribution of the article with the strict proviso that no changes or edits are made and the original work is properly cited (including links to both the formal publication through the relevant DOI and the license). See: https://creativecommons.org/licenses/by-nc-nd/4.0/.

\section{References}

1. Jurado J, Javidfar J, Newmark A, et al. Minimally invasive thymectomy and open thymectomy: outcome analysis of 263 patients. Ann Thorac Surg 2012;94:974-81; discussion 981-2.

2. Hess NR, Sarkaria IS, Pennathur A, et al. Minimally invasive versus open thymectomy: a systematic review of surgical techniques, patient demographics, and perioperative outcomes. Ann Cardiothorac Surg 2016;5:1-9.

3. Lewis RJ, Caccavale RJ, Sisler GE. Imaged thoracoscopic surgery: a new thoracic technique for resection of mediastinal cysts. Ann Thorac Surg 1992;53:318-20.

4. Wu CF, Gonzalez-Rivas D, Wen CT, et al. Single-port video-assisted thoracoscopic mediastinal tumour resection. Interact Cardiovasc Thorac Surg 2015;21:644-9.

5. Li Q, Sihoe A, Wang H, et al. Short-term outcomes of single- versus multi-port video-assisted thoracic surgery in mediastinal diseases. Eur J Cardiothorac Surg 2018;53:216-20.

6. Xie D, Wang H, Fei K, et al. Single-port video-assisted thoracic surgery in 1063 cases: a single-institution experience. Eur J Cardiothorac Surg 2016;49 Suppl 1:i31-6.

7. Yoshino I, Hashizume M, Shimada M, et al. Thoracoscopic thymomectomy with the da Vinci computer-enhanced surgical system. J Thorac 
Cardiovasc Surg 2001;122:783-5.

8. Yim AP, Kay RL, Ho JK. Video-assisted thoracoscopic thymectomy for myasthenia gravis. Chest 1995;108:1440-3.

9. Agatsuma H, Yoshida K, Yoshino I, et al. Video-Assisted Thoracic Surgery Thymectomy Versus Sternotomy Thymectomy in Patients With Thymoma. Ann Thorac Surg 2017;104:1047-53.

10. Pennathur A, Qureshi I, Schuchert MJ, et al. Comparison of surgical techniques for early-stage thymoma: feasibility of minimally invasive thymectomy and comparison with open resection. J Thorac Cardiovasc Surg 2011;141:694-701.

11. Burt BM, Yao X, Shrager J, et al. Determinants of Complete Resection of Thymoma by Minimally Invasive and Open Thymectomy: Analysis of an International Registry. J Thorac Oncol 2017;12:129-36.

12. Rocco G, Martin-Ucar A, Passera E. Uniportal VATS wedge pulmonary resections. Ann Thorac Surg 2004;77:726-8.

13. Guido-Guerrero W, Bolaños-Cubillo A, GonzálezRivas D. Single-port video-assisted thoracic surgery (VATS)-advanced procedures \& update. J Thorac Dis 2018;10:S1652-61.

14. Ashrafian H, Clancy O, Grover V, et al. The evolution of robotic surgery: surgical and anaesthetic aspects. Br J Anaesth 2017;119:i72-i84.

Cite this article as: Zeng L, Wang W, Han J, Zhu L, Zhao J, Tu Z. Uniportal video-assisted thoracoscopic surgery and robot-assisted thoracoscopic surgery are feasible approaches with potential advantages in minimally invasive mediastinal lesions resection. Gland Surg 2021;10(1):101-111. doi: 10.21037/ gs-20-536
15. Buentzel J, Heinz J, Hinterthaner M, et al. Robotic versus thoracoscopic thymectomy: The current evidence. Int J Med Robot 2017. doi: 10.1002/rcs.1847.

16. Qian L, Chen X, Huang J, et al. A comparison of three approaches for the treatment of early-stage thymomas: robot-assisted thoracic surgery, video-assisted thoracic surgery, and median sternotomy. J Thorac Dis 2017;9:1997-2005.

17. O'Sullivan KE, Kreaden US, Hebert AE, et al. A systematic review of robotic versus open and video assisted thoracoscopic surgery (VATS) approaches for thymectomy. Ann Cardiothorac Surg 2019;8:174-93.

18. Rocco G, Brunelli A, Jutley R, et al. Uniportal VATS for mediastinal nodal diagnosis and staging. Interact Cardiovasc Thorac Surg 2006;5:430-2.

19. Akter F, Routledge T, Toufektzian L, et al. In minor and major thoracic procedures is uniport superior to multiport video-assisted thoracoscopic surgery? Interact Cardiovasc Thorac Surg 2015;20:550-5.

20. Gonzalez-Rivas D, Wu CF, de la Torre M. Uniportal video-assisted thoracoscopic thymectomy and resection of a giant thymoma in a patient witness of Jehova. J Thorac Dis 2017;9:E556-9.

21. Deng J, She Y, Zhao M, et al. Simultaneously thoracoscopic resection of lung cancer and anterior mediastinal lesions by video-assisted thoracoscopic surgery. Ann Transl Med 2019;7:333. 


\section{Supplementary}

Table S1 Regression analysis of the risk factors for adverse surgical outcomes

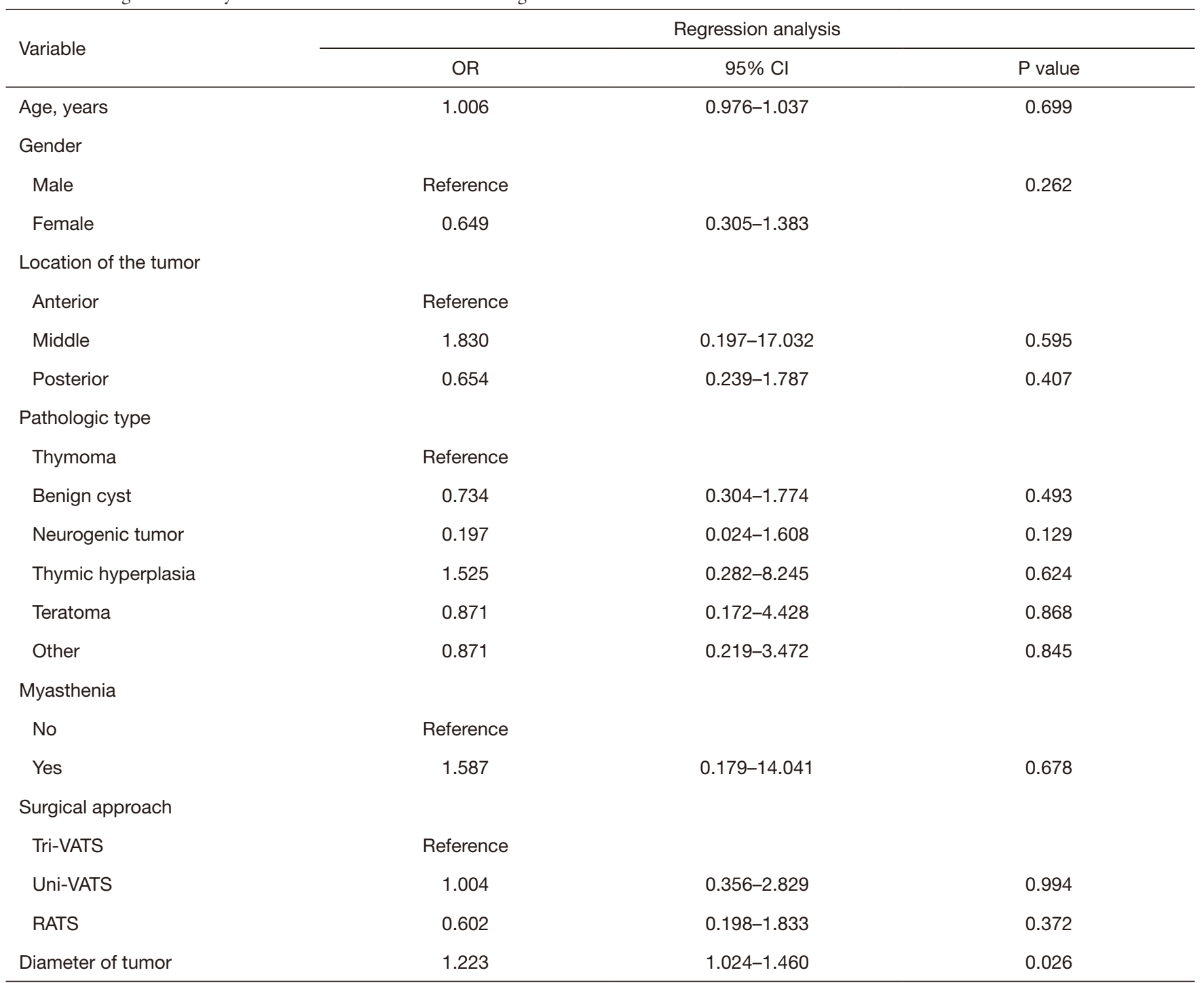

Cl, confidence interval; OR, odds ratio; RATS, robot-assisted thoracic surgery; Tri-VATS, triportal video-assisted thoracoscopic, Uni-VATS, uniportal video-assisted thoracoscopic. 
Table S2 Clinicopathological characteristics of the study population after PSM

\begin{tabular}{|c|c|c|c|c|c|c|}
\hline Characteristics & Tri-VATS $(n=40)$ & Uni-VATS $(n=40)$ & $P$ value & VATS group $(n=49)$ & RATS group $(n=49)$ & $P$ value \\
\hline Diameter, mediate (min, max), cm & $2.3(1.0,7.0)$ & $2.4(1.2,7.2)$ & 0.272 & $2.6(1.0,7.0)$ & $2.5(0.8,7.5)$ & 0.935 \\
\hline Gender, n (\%) & & & 0.654 & & & 0.840 \\
\hline Male & $20(50.0)$ & $18(45.0)$ & & $25(51.0)$ & $24(49.0)$ & \\
\hline Location, n (\%) & & & 0.233 & & & 0.288 \\
\hline Anterior & $26(65.0)$ & $22(55.0)$ & & $38(77.6)$ & $39(79.6)$ & \\
\hline Middle & $0(0.0)$ & $3(7.5)$ & & $0(0.0)$ & $2(4.1)$ & \\
\hline Posterior & $14(35.0)$ & $15(37.5)$ & & $11(22.4)$ & $8(16.3)$ & \\
\hline No & $40(100.0)$ & $40(100.0)$ & & $48(98.0)$ & $48(98.0)$ & \\
\hline Pathologic type, n (\%) & & & 0.361 & & & 0.744 \\
\hline Thymoma & $8(20.0)$ & $4(10.0)$ & & $14(28.6)$ & $11(22.4)$ & \\
\hline Benign cyst & $15(37.5)$ & $21(52.5)$ & & $22(44.9)$ & $26(53.1)$ & \\
\hline Neurogenic tumor & $8(20.0)$ & $7(17.5)$ & & $7(14.3)$ & $4(8.2)$ & \\
\hline Thymic hyperplasia & $3(7.5)$ & $0(0.0)$ & & $3(6.1)$ & $2(4.1)$ & \\
\hline Teratoma & $2(5.0)$ & $2(5.0)$ & & $1(2.0)$ & $2(4.1)$ & \\
\hline Other & $4(10.0)$ & $6(15.0)$ & & $2(4.1)$ & $4(8.2)$ & \\
\hline
\end{tabular}

CM, centimeter; RATS, robot-assisted thoracic surgery; SD, standard deviation; Tri-VATS, triportal video-assisted thoracoscopic, UniVATS, uniportal video-assisted thoracoscopic. 
Table S3 Regression analysis of the risk factors for adverse surgical outcomes after propensity score-matched analysis between the VATS group and the RATS group

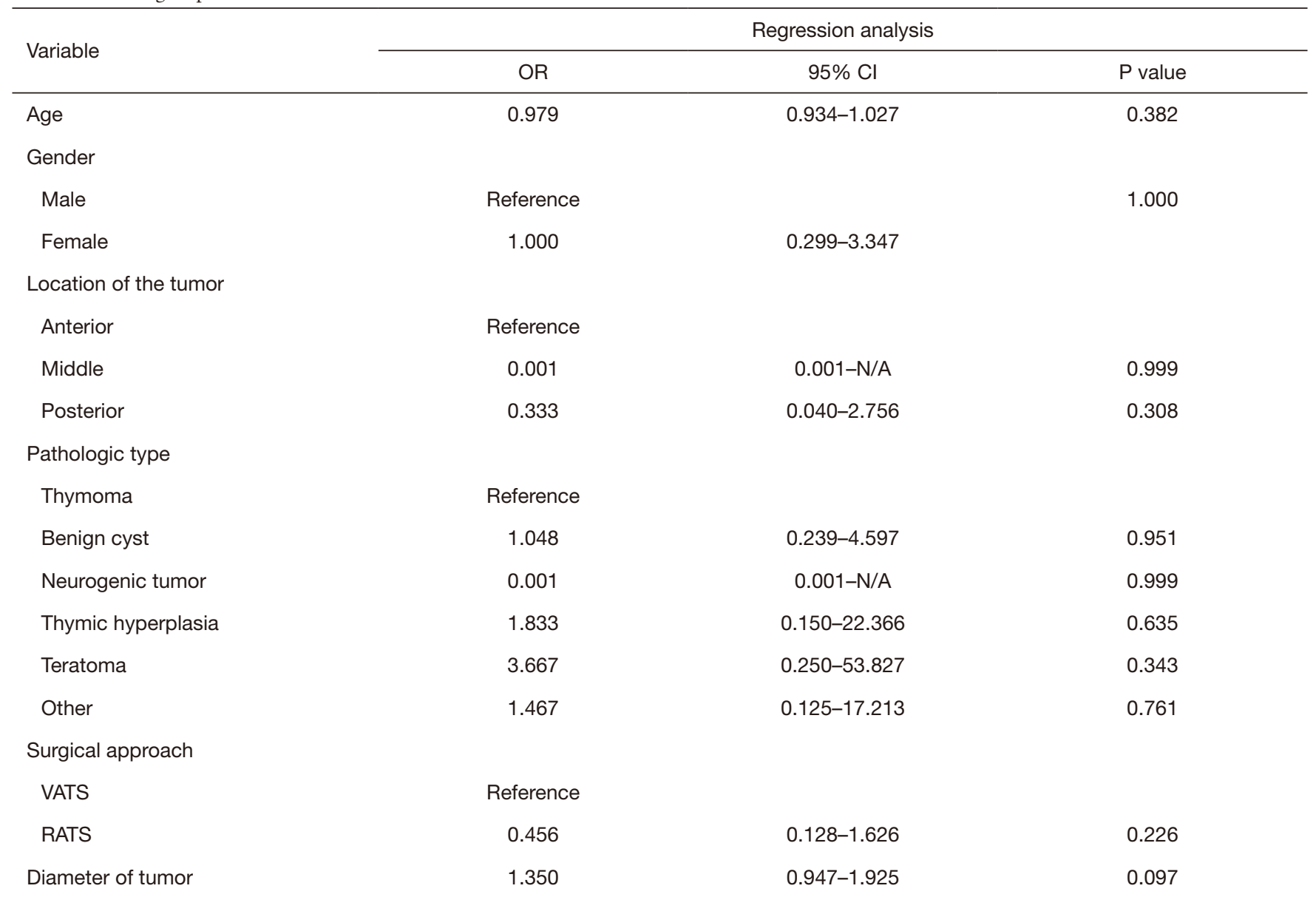

CI, confidence interval; OR, odds ratio; RATS, robot-assisted thoracic surgery; Tri-VATS, triportal video-assisted thoracoscopic, Uni-VATS, uniportal video-assisted thoracoscopic; N/A, not applicable. 
Table S4 Regression analysis of the risk factors for adverse surgical outcomes after propensity score-matched analysis between the tri-VATS group and the uni-VATS group

\begin{tabular}{|c|c|c|c|}
\hline Variable & \multicolumn{3}{|c|}{ Regression analysis } \\
\hline Age & 0.998 & 0.930-1.072 & 0.962 \\
\hline \multicolumn{4}{|l|}{ Gender } \\
\hline Male & Reference & & 0.233 \\
\hline \multicolumn{4}{|l|}{ Location of the tumor } \\
\hline Anterior & Reference & & \\
\hline Middle & 0.343 & $0.004-29.007$ & 0.637 \\
\hline Posterior & 4.540 & $0.565-36.500$ & 0.155 \\
\hline Benign cyst & 0.206 & $0.022-1.940$ & 0.168 \\
\hline Neurogenic tumor & 0.064 & $0.003-1.407$ & 0.081 \\
\hline Thymic hyperplasia & 3.376 & $0.143-79.720$ & 0.451 \\
\hline Teratoma & 2.126 & $0.073-61.666$ & 0.661 \\
\hline Other & 0.347 & $0.023-5.138$ & 0.442 \\
\hline \multicolumn{4}{|l|}{ Surgical approach } \\
\hline Tri-VATS & Reference & & \\
\hline Uni-VATS & 0.673 & $0.194-2.333$ & 0.533 \\
\hline
\end{tabular}

$\mathrm{Cl}$, confidence interval; OR, odds ratio; RATS, robot-assisted thoracic surgery; Tri-VATS, triportal video-assisted thoracoscopic, Uni-VATS, uniportal video-assisted thoracoscopic. 